\title{
Construction Technique and Temperature Control of Building Environment Based on Thermal Process Model
}

\author{
Xiuli $\mathrm{Wu}^{1, \mathrm{a}}$, Yaru Fan ${ }^{2, \mathrm{~b}}$ \\ ${ }^{1}$ Wuhan University of Technology Huaxia College, Wuhan, 430074, China; \\ ${ }^{2}$ Wuhan Zhongwei Estate, Wuhan, 430050, China \\ ahbwj1976@163.com, ${ }^{\text {b373236719@qq.com }}$
}

Keywords: Spiral staircase; CAD accurate modeling; State space equation; Thermal process mode; Building environment design

\begin{abstract}
To solve the problems regarding the construction technology of $360^{\circ}$ large spiral staircase and improve the quality of framework supports, the frequency for common failings for the quality of spiral staircase decreases from $14 \%$ to $5 \%$ according to the spiral curve equation and by applying CAD software accurate modeling and tracking control for highly precise DS1 level. Meanwhile, the construction cost is significantly reduced and a new and mature construction technology for spiral staircase is formed, which can be taken as reliable reference for the relevant construction afterwards.
\end{abstract}

\section{Introduction}

Some certain project in Donghu is frame structure. One casting reinforced concrete $360^{\circ}$ spiral staircase is designed from the basement floor to the ground floor with $5.4 \mathrm{~m}$ high, diameter of $1.7 \mathrm{~m}$ in outer arc of the floor, $1.2 \mathrm{~m}$ wide and $0.5 \mathrm{~m}$ inner arc of the staircase. There are altogether 29 steps and each step is $186 \mathrm{~mm}$ high; length of inner arc is $125 \mathrm{~mm}$ while outer arc $426 \mathrm{~mm}$ and $170 \mathrm{~mm}$ thickness of gang-board. The gang-board of the staircase are continuous casting and the inner side of the back strap is penetrated into the $300 \mathrm{~mm}$ shear wall while its outer side is penetrated into the stairway beams of the outer gang-board. Since there is shear wall, changeable gang-board and stairway beams in the designing of the staircase, therefore, the construction and framework construction is quite complicated in this staircase.

\section{Curve equation for spiral staircase}

The thermal process solution uses a state space equation method, which is different from finite difference method, however state space method can achieve the spatial difference, and keep the time continuity, which is very useful for time process analysis of many spaces. In state space equation, the internal temperature uniformity simulation objects need to handle separately, each is as a temperature point, however the interior temperature is similar simulated object as a temperature points, while are the function of time, so that it can be these temperature joint cubic equations, to generate of state space vector, using matrix algebraic solution.

In order to reduce the order of equation, to simplify equations, the wall thermal process is treated as a first-order process, the indoor air temperature is lumped into a single node, and it is assumed that the wall material properties do not change in a certain period of time. If the window temperature time continuous, so the wall enclosure will carry out space discrete time that does not produce large errors. Considering the outdoor high frequency disturbance, the sampling interval of outdoor perturbations is set to $1 \mathrm{~h}$. Suppose further that the building various disturbances are independent of room air temperature, which is known quantity before calculating room temperature. For the relationships between air conditioning system heat disturbance and spatial temperature, the relationships between heat exchange of natural ventilation and temperature, the relationships between adjacent room ventilation heat and adjacent room temperature, they need to be solved through the establishment of joint equation.

The heat convection, indoor unit, air exchange as well as air conditioning system and the heat 
exchange quantity of building space are separated from air temperature equation, which can get:

$$
\begin{aligned}
& t_{a}(\tau)=\sum_{i} e^{\lambda_{i} \Delta \tau} t_{a i}(\tau-\Delta \tau)+\sum_{k}\left[\Phi_{k, l} u_{k}(\tau-\Delta \tau)+\Phi_{k, 0} u_{k}(\tau)\right]+ \\
& \sum_{j} \Phi_{j, 1} t_{j}(\tau-\Delta \tau)+\sum_{j} \Phi_{j, 0} t_{j}(\tau)+\Phi_{\text {hvac }} q_{\text {hvac }}+\Phi_{\text {hvac }} c_{p} \rho G_{\text {out }}\left[t_{\text {out }}(\tau)-t_{\mathrm{a}}(\tau)\right]+ \\
& \sum_{j} \Phi_{\text {hvac }} c_{p}(\tau) \rho G_{j}\left[t_{j}(\tau)-t_{\mathrm{a}}(\tau)\right]
\end{aligned}
$$

In formula (1), $\Phi \mathrm{k}, 1$ and $\Phi \mathrm{k}, 0$ are respectively the previous time and disturbance coefficient of the indoor air temperature of the current time after thermal disturbances of the role of separation; $\Phi \mathrm{j}, 1$ and $\Phi \mathrm{j}, 0$ are expressed as $\mathrm{j}$ adjacent chamber that is respectively the previous time and current time for the indoor air temperature perturbation coefficient; hvac is the air conditioning system disturbance on the disturbance factor of indoor air temperature. It is credited as:

$$
\begin{aligned}
& t b z(\tau)=\sum_{i} e^{\lambda_{i} \Delta \tau} t_{a i}(\tau-\Delta \tau)+\sum_{k}\left[\Phi_{k, l} u_{k}(\tau-\Delta \tau)+\Phi_{k, 0} u_{k}(\tau)\right]+ \\
& \sum_{j} \Phi_{j, 1} t_{j}(\tau-\Delta \tau)
\end{aligned}
$$

In formula (2), tbz ( $\tau$ ) is said the room air temperature, which is regardless of the current states heat convection, indoor unit, air exchange and the heat exchange of building space

The room temperature can be expressed as:

$$
\begin{aligned}
& t_{a}(\tau)=t b z(\tau)+\sum_{j} \Phi_{j, 0} t_{j}(\tau)+\Phi_{\text {hvac }} q_{\text {hvac }}+\Phi_{\text {hvac }} c_{p} \rho G_{\text {out }}\left[t_{\text {out }}(\tau)-t_{\mathrm{a}}(\tau)\right]+ \\
& \sum_{j} \Phi_{\text {hvac }} c_{p}(\tau) \rho G_{j}\left[t_{j}(\tau)-t_{\mathrm{a}}(\tau)\right]
\end{aligned}
$$

In formula (3), it is known tbz condition, heat convection, indoor unit, air exchange, air conditioning system and heat exchange quantity of building space, which is the resolution of indoor building temperature estimation, building load calculation, and basic expressions of air conditioning system simulation.

After having basic expressions, hypotheses building room number is $\mathrm{n}$, the $\mathrm{K}$ room temperature expression is carried out merge similar items, such as a simplified formula:

$$
a_{k k} t_{k}(\tau)+\sum_{j} a_{k j}(\tau) t_{j}(\tau)+b_{k k}(\tau) q_{h v a c, k}(\tau)=c_{k}(\tau)
$$

All n room temperature expressions of simultaneous equation can be:

$$
A^{\prime}+B^{\prime} Q=C^{\prime}
$$

In formula (5), $\mathrm{T}, \mathrm{Q}$ and $\mathrm{C}^{\prime}$ are expressed as $\mathrm{n}$-dimensional vector, $\mathrm{A}^{\prime}$ and $\mathrm{B}^{\prime}$ are $\mathrm{n} \times \mathrm{n}$ matrix, $T$ is temperature vector, $Q$ is air conditioning heat consumption vector, $A^{\prime}$ is temperature node matrix, $\mathrm{B}^{\prime} \quad$ is air-conditioning system on the temperature effect of the diagonal matrix, $\mathrm{C}^{\prime}$ is all known composition of vector, so it can be solved by using matrix algebra.

\section{Technique Process and Operation Methods}

Construction Line-putting of the Staircase. Spiral staircase is made up by the fraction of the helicoid composed by the inner and outer helicoid with different diameters [1], which radiates to the outside from the center in each step. Though inner and outside of the width of the steps are different, but the elevation in the inner and outer side of the helicoid is quite the same. The construction line-putting of the spiral staircase is comparatively complex. By applying the CAD drawing, the coordinate of the control-point of the beam can be derived and can pop-up the sideline of the beam as the control line in the field. The line-putting graph of the staircase positioning is as follows: 


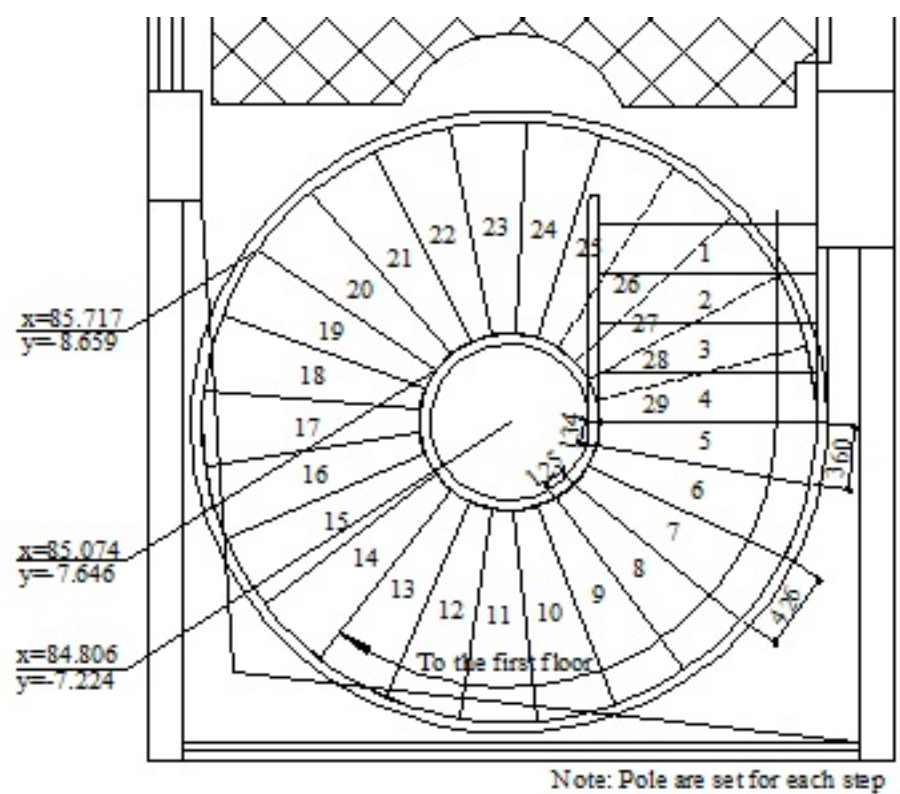

Fig. 1 The line-putting layout for the measurement of spiral staircase

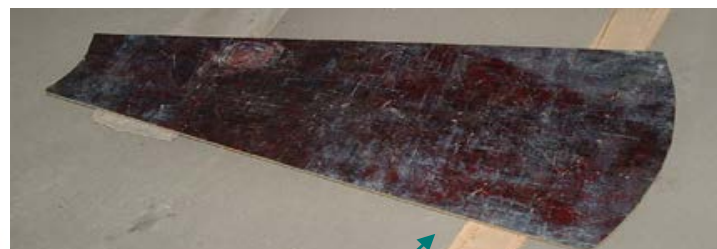

Fig. 2 Sampling of the stereoty framework of the staircase

Lofting for the Spiral Staircase. According to the line-putting layout for the measurement of spiral staircase, the framework blanking adopts the CAD technology to simulate the lofting. [2] In the following figure, the geometric dimension of the blanking is determined. The center, inner and outer arc of the inner and outer circles are positioned by total station device and labeled clearly by oil painting. Foot-board line of the staircase is only printed from the inner arc to the outer arc which is also labeled by red oil painting. The stepping line can directly be equally divided by the measurement.

Equally Divided the Inner and Outer. According to the center of the spiral staircase, the inner and outer arc of the staircase is given. According to the dimension of the spiral staircase, it is divided equally into several parts and each part is the base point of the column of the form board and the real height of each upright column can be calculated.

Setting Up the Support for Staircase framework. According to the property of the features of the spiral staircase, the inner and outer upright is equal in each radioactive rays of the arc [3]. Several upright columns in the same radioactive rays should be arranged into the form of bent column. Vertically arrange steel tube in the same side of the inner or outer arc of each stepping line and the distance between upright steel tube and stepping line should be $24 \mathrm{~mm}$ (half of the 48 steeling tube) and add bent column in half the width of the outer staircase. Connect the erected tube with horizontal bar and set up bottom horizontal tube and cross bridging to enhance stiffness and ensure that the upright tube would not in sideways or skew. Copy the corresponding height of the bottom of the staircase by the stepping line and labeled it out with red paint. Then set up the horizontal steel tube to make the upper of the tube in accordance with the elevation printed by red paint in corresponding upright column. The inner arc length of the upright column is $5 \mathrm{~cm}$ which is $10 \mathrm{~cm}$ far from the outer arc. Calculate the accurate elevation of each step of the staircase by CAD simulation and label the elevation on each inner or outer column by laser level. Check the two ends of the horizontal tube by suspension wire. If the center line of the horizontal tube coincides with the stepping line of the lower sample, the elevation is in accordance with the calculation. Otherwise, the elevation is inaccurate and should be adjusted until it is accurate, as is shown in the following,as Figure 3 shown. 


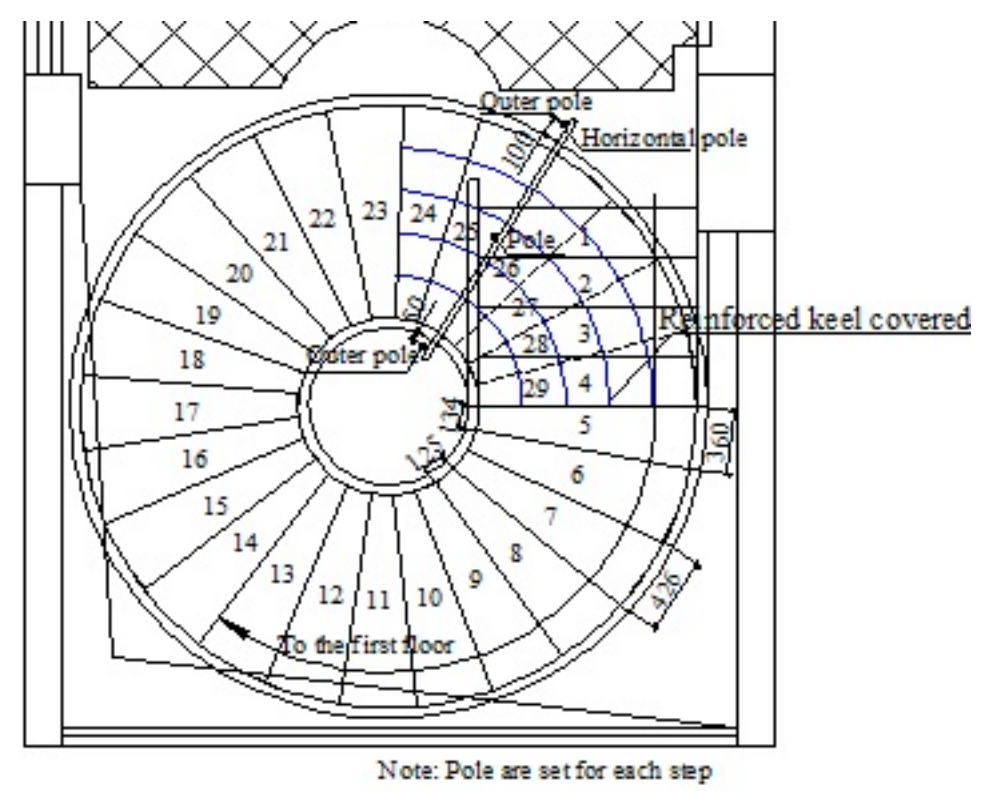

Fig.3 The supporting layout for the spiral staircase

Setting Up the Staircase framework. Arrange the horizontal steel tube closely clinging to the steps according to the direction of spirals and arrange the steel bar $\varphi 14 @ 150$ vertically and fasten with iron wires in the cross of the reinforced steel bar and the horizontal steel tube. Cut evenly by electric welding for the parts of the steel bar beyond the platform of the staircase so as not to affect the erecting molding.

On the arranged $\varphi 14$ steel bar, fasten $50 * 50$ wooden square-column on the vertically $\varphi 14$ steel bar direction and the gap is 400 . Arrange the base plate of $10 \mathrm{~mm}$ nine splint (whose form is wedge shaped plate wider in outside and narrow in inside) made in advance by the large end towards the outside and small end towards the inside (center). Put them on the wood and tighten them from the starting of the staircase and drill 2 holes in the base plate; use the No. 14 iron wire to fasten the $\varphi 14$ steel keel, thus forming the baseboard of the staircase with a slippery twisted helicoid.

Therefore, in arranging the height of the upright column, special attention should be paid that the thickness of the square woods, baseboard and the arc steel bar should be reduced, by this way; the height of the upright column can be accurate. This example takes the height of the square woods with $50 \mathrm{~mm}$, thickness of the baseboards of $10 \mathrm{~mm}$ and steel bar of $14 \mathrm{~mm}$ so that totally $74 \mathrm{~mm}$ should be reduced, adding with the thickness of the thickness of the floor which is $350 \mathrm{~mm}$, therefore, altogether $424 \mathrm{~mm}$ should be deducted.

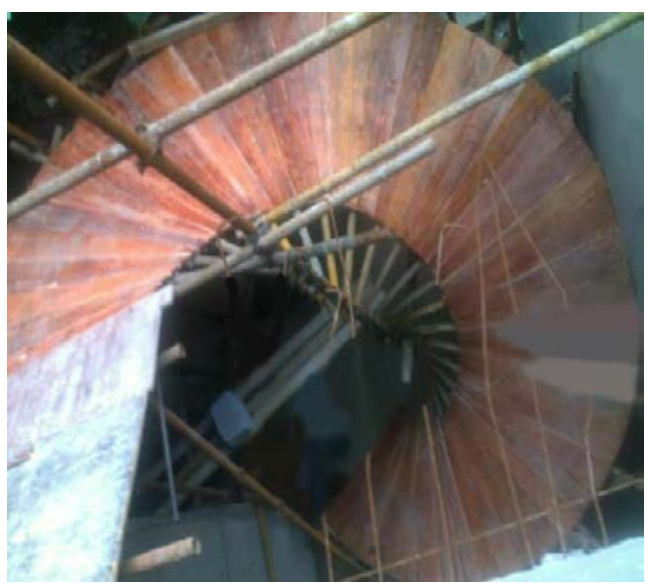

Fig. 4 The support picture of the spiral staircase

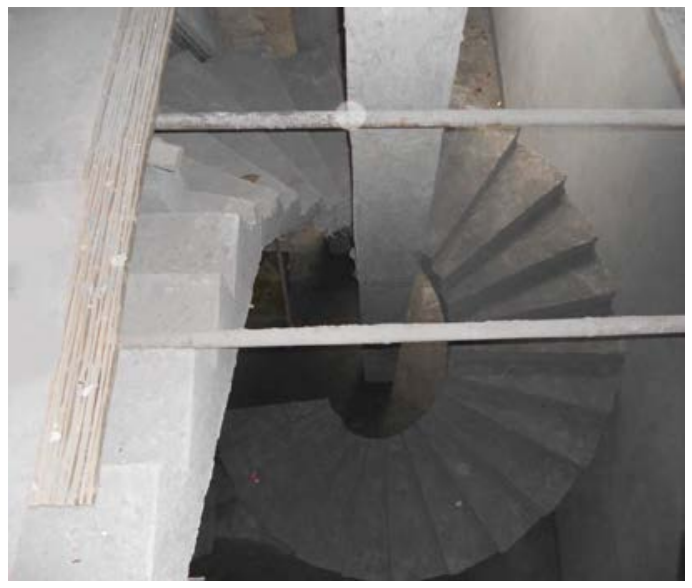

Fig. 5 Realistic picture of the spiral staircase

The twisted helicoid is to fasten the steel bar of the structure of the staircase on the bottom die according to the planning. The side forms should be installed in the inner and outer arc and the dimension; arc, position and elevation of the blanking should be accurately controlled and rechecked with wire weight. After adjustment, kick board plate should be installed and then install 
the side forms of the staircase beams which is supported by wood beam in the side. The stepping board is support by wood and then casts concrete on the staircase and keeps maintenance (Fig. 5).

\section{Quality Control}

Spiral staircase is circular curve that varies in spatial situation and the basic principle for the changes in curve should be mastered. The quantity for equally division should base on the length of the arc and satisfy the requirements of the continuous slippery baseboard for supporting the framework, then calculate appropriately and then the accurate lofting data will be obtained.

Use CAD to calculate the dimension of the blanking of the stepping modeling so that the gap for installation will not be too big[4]. Recheck the dimension of positioning and elevation by total station device and level after positioning.

Whether the concrete place after framework is in accordance with the place of lofting should be checked and pay attention that the installation of the framework should have enough intensity and stiffness [5].

Check the distance and position of the opposite column and horizontal column according to the requirement of the framework support. Set up according to the strict requirements of designed dimension; the connector of the upright column and horizontal column should be staggered to make sure that the vertical deviation of the upright column less than $15 \mathrm{~mm}$ and the horizontal deviation of the horizontal level less than 20mm [6].

The first is a natural room temperature analysis that is defined room temperature condition under no air conditioning control system. Natural room temperature disturbance and delay reflect the buildings other differences in the ventilation, heat exchange, and indoor thermal disturbance and toward. Figure 6 shows the theme pavilion in natural room temperature curve of indoor radial ends (temperature 1 and temperature 3 ) and the middle part ( temperature 2 ).

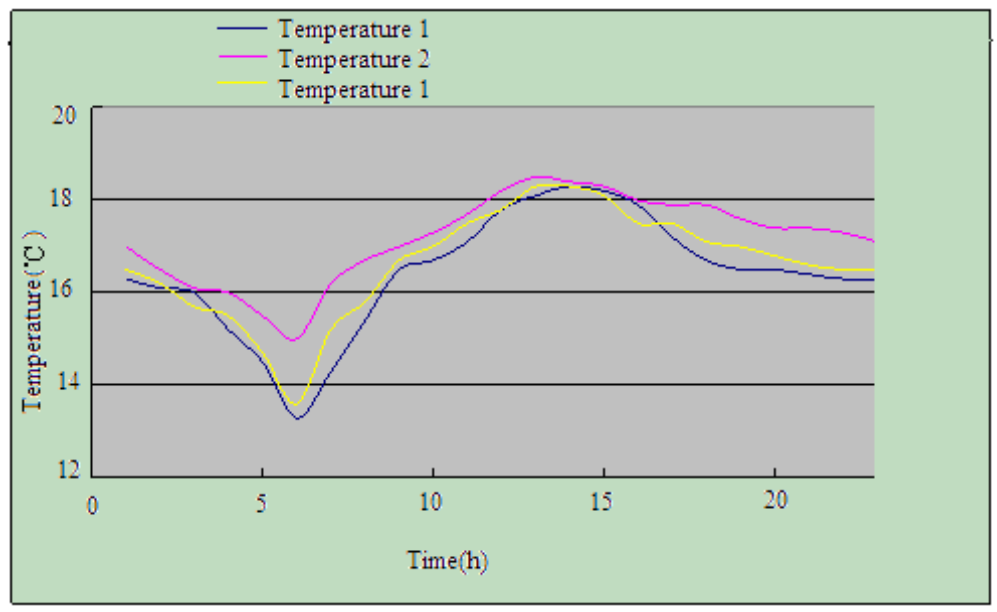

Fig.6 Theme Pavilion indoor natural room temperature curve

The second is the building load calculation that is to be inside a building temperature control in a certain range, air conditioning system provides cold heat in unit time. Through the proper consideration of the influence of various factors, it can accurately calculate the building cold, heat load. This will help with the air-conditioning system design and realize the optimal allocation of resources. Air-conditioning system's control principle is: in heating condition, temperature control is the upper limit of air conditioning system; during the transition, the air conditioning load is 0 , the buildings are also able to maintain the temperature in a certain scope,as shown in Figure 7.

Through the analysis, people can find that the buildings surrounding use a larger capacity ratio of materials that can effectively use of solar energy and indoor heat savings, and can also use the advantage of the surrounding environment, such as water resources and so on, which can maintain the relatively balance purpose of indoor building temperature. The outdoor air is leaded into the interior by the underground ventilation facilities, because of the use of geothermal energy, which can be warm in winter and cool in summer, it saves the energy consumption. Thus, people can confirm science and art, humanity and intelligence for the architectural design that is very 
important.

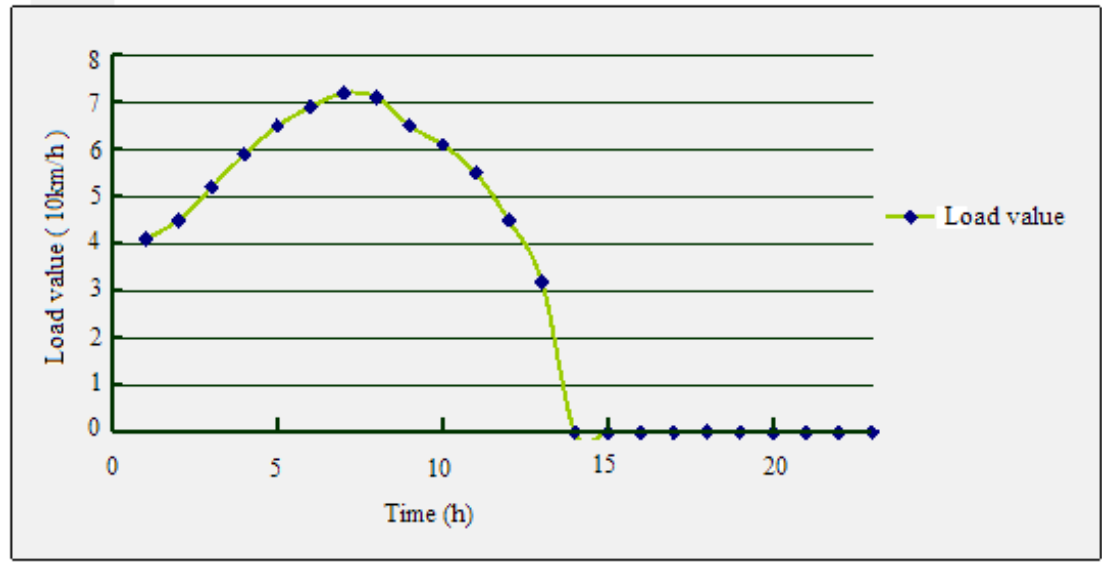

Fig.7 Indoor temperature control load curve

\section{Conclusion}

Construction by applying the above mentioned spiral staircase can avoid the set-shaped steel framework; it can save 20,000 Yuan RMB for material expense, 5,000 RMB for construction expense; reduce 10,000 RMB for labor cost, which is 35,000RMB in total. Beside, the speed for framework is fast; the curve is slippery and accurate; the construction quality is easy to control and can meet the requirements for designing and relevant acceptance specification.Meanwhile, a new and mature construction technology for spiral staircase is formed, which can be taken as reliable reference for the relevant construction afterwards.

\section{Reference}

[1]Chen Feng, Deng Yuchun, Xue Zhifeng, Wu Ruhong. Building environment simulation toolpack DeST. Heating ventilating \& air conditioning, 2009 (29): 58-63.

[2]Gao Qinhe, Wang Sunan. Nonlinear dynamic system identification based on elman neural network [J]. Computer engineering and applications, 2007, 43 (31):87-89.

[3]Si Fengqi, Hong Jun, Xu Zhigao. Application of dynamic system based on improved Elman neural network. Measurement test method [J]. Journal of southeast university: natural science edition, 2009, 35 (1) :50-54

[4]Jin Zhaofen, Zhu Yingxin. Construction environmental science [M]. Beijing: China construction industry press, 2010:136-146.

[5]Jian Yiwen, Jiang Yi. Consistency analysis of residential room temperature simulation and measured data [J]. esRidential science and technology, 2009 (7): 35-38.

[6]Xu Congzhi, Li Guiwen. Accurate designs required for houses and environments [J]. Journal of Harbin institute of technology, 2009,35(3): 87-89.

[7]Guo Yijun. Low carbon ecological of construction of environment design [D]. Taiyuan polytechnic university, 2010:2-15.

[8]Liu Chunfu. Analysis of the small scale water conservancy construction and environmental design [J]. Journal of Nanchang University, 2012(16): 75-79. 